\title{
On the Invariant Sets of a Family of Quadratic Maps
}

\author{
M. F. Barnsley ${ }^{\star}$ J. S. Geronimo ${ }^{\star}$, and A. N. Harrington \\ School of Mathematics, Georgia Institute of Technology, Atlanta, GA 30332, USA
}

\begin{abstract}
The Julia set $B_{\lambda}$ for the mapping $z \rightarrow(z-\lambda)^{2}$ is considered, where $\lambda$ is a complex parameter. For $\lambda \geqq 2$ a new upper bound for the Hausdorff dimension is given, and the monic polynomials orthogonal with respect to the equilibrium measure on $B_{\lambda}$ are introduced. A method for calculating all of the polynomials is provided, and certain identities which obtain among coefficients of the threeterm recurrence relations are given. A unifying theme is the relationship between $B_{\lambda}$ and $\lambda$-chains $\lambda \pm V\left(\lambda \pm V(\lambda \pm \ldots)\right.$, which is explored for $-\frac{1}{4} \leqq \lambda \leqq 2$ and for $\lambda \in \mathbb{C}$ with $|\lambda| \leqq \frac{1}{4}$, with the aid of the Böttcher equation. Then $B_{\lambda}$ is shown to be a Hölder continuous curve for $|\lambda|<\frac{1}{4}$.
\end{abstract}

\section{Introduction}

In this paper we consider the Julia set $B_{\lambda}$ for the mapping

$$
T_{\lambda} z=(z-\lambda)^{2}, \quad z \in \mathbb{C},
$$

of the complex plane into itself, where $\lambda$ is a parameter which may be real or complex. Here $T_{\lambda}$ is equivalent to $z \rightarrow 1-\lambda z^{2}$ which has been studied in the context of iterated maps of intervals, see $[10,13]$, and also to $z \rightarrow z^{2}+\lambda$, see $[11]$.

$B_{\lambda}$ was first studied by Fatou [12] and Julia [19] in the context of arbitrary rational transformations. With the notation

$$
T_{\lambda}^{0} z=z, \text { and } T_{\lambda}^{n+1} z=T_{\lambda}\left(T_{\lambda}^{n} z\right) \text { for } n \in\{1,2,3, \ldots\},
$$

$B_{\lambda}$ can be defined to be those points in $\mathbb{C}$ where $\left\{T_{\lambda}^{n} z\right\}$ is not normal. This is the starting point of the survey by Brolin [8]. Equivalently $B_{\lambda}$ can be defined to be the closure of the set of all repulsive $k$-cycles, $k \in\{1,2,3, \ldots\},[12]$. This shows at once the relevance of $B_{\lambda}$ to the corresponding iterated real map where $B_{\lambda} \cap \mathbb{R}$ plays a central role.

* Supported by NSF Grant MCS-8104862

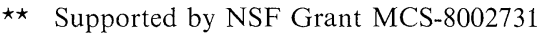


Our approach is to consider the set $\tilde{B}_{\lambda}$ of formal objects which we call $\lambda$-chains, $\{\lambda \pm V(\lambda \pm V(\lambda \pm \ldots\}$, where all half-infinite sequences of plus and minus signs are included, and where the branch cut is fixed, for example, on the negative real axis. We use $\lambda$-chains as a unifying idea in our discussion of $B_{\lambda}$. For $\lambda>2$ we easily work out a one-to-one correspondence between the elements of $\tilde{B}_{\lambda}$ and the points of $B_{\lambda}$. For $-\frac{1}{4} \leqq \lambda \leqq 2$ the correspondence is exhibited via the Böttcher equation and conformal mapping, and for some values of $\lambda$ we show only that almost all $\lambda$-chains correspond to individual points in $B_{\lambda}$.

In Sect. 2.1 we examine the case $2 \leqq \lambda<\infty$, where we give a direct construction of $B_{\lambda}$ which displays its connection with $\tilde{B}_{\lambda}$. Use is made of a distance function, natural to $B_{\lambda}$, which yields a simple demonstration that the Lebesgue measure of $B_{\lambda}$ is zero for $\lambda>2$ and which provides a new upper bound on its Hausdorff dimension. We also construct the equilibrium measure $\sigma$ on $B_{\lambda}$ by means of a special sequence of approximating measures. The latter are related to the monic orthogonal polynomials with respect to $\sigma$ which are considered in Sect. 2.2. A method for calculating all of the polynomials is provided, and certain identities which obtain among the coefficients of the three-term recurrence relations are discovered. Interrelations between the polynomials reflect the structure of $B_{\lambda}$. In particular, it is found possible to describe completely an infinite subsequence of Padé approximants to the generating function. The polynomials generalize those of Tchebycheff, to which they are simply related when $\lambda=2$.

In Sect. 3.1, we present for the case $-\frac{1}{4} \leqq \lambda \leqq 2$ two constructions for $B_{\lambda}$; one from the "outside" and one from the "inside". Each involves a sequence of functions which converges to the solution of the functional equation $F_{\lambda}(z)$ $=\lambda+\sqrt{F_{\lambda}\left(z^{2}\right)}$ with $F_{\lambda}(z)=z+O(1)$ at $\infty$. Here $F_{\lambda}$ maps the exterior of the unit disk conformally onto a region bounded by $B_{\lambda}$, and by means of $F_{\lambda}$ we relate $\lambda$-chains to $B_{\lambda}$. The first construction involves the formation of an increasing sequence of domains, successive inverse images under $T_{\lambda}$ of a neighborhood of $\infty$, as suggested by Fatou and by Julia. We include it for completeness, and to shorten the proof of the second construction which involves a decreasing sequence of domains. Of interest are the complements of the domains which form an increasing sequence of trees and describe $B_{\lambda}$ from the interior. We have recently proved [4] that this sequence of trees converges to $B_{\lambda}$ itself for infinitely many values of $\lambda \in(0,2)$.

In Sect. 3.2 we begin by restricting attention to $0 \leqq \lambda \leqq 0.2$ and we make explicit calculations with $\lambda$-chains to show that they are well-defined and that $F_{\lambda}(z)$ can be extended continuously to the unit circle. Next we make analytic extensions to $\lambda$ in the set $L=\{\lambda \in \mathbb{C}|| \lambda \mid<1 / 4\}$ and show that the mappings $G_{\lambda}(\theta)=F_{\lambda}\left(e^{i \theta}\right)$ are uniformly Hölder continuous for $\lambda$ in any compact subset of $L$.

In Sect. 4 we give a few pictures in connection with $B_{\lambda}$.

\section{The Case $2 \leqq \lambda<\infty$}

\subsection{Construction of $B_{\lambda}$ and of an Invariant Measure}

Throughout this section we assume $\lambda \in[2, \infty)$. We begin with a construction of the Julia set $B_{\lambda}$ which displays its connection with $\tilde{B}_{\lambda}$. Thus we obtain a concrete 
example of the correspondence apparently first introduced by Fatou [12, Sect. 23] for arbitrary rational transformations. Our approach uses a new distance function which allows us to obtain a significant improvement on Brolin's estimate [8, Theorem 12.2] of the Hausdorff dimension of $B_{\lambda}$.

We conclude this section with the construction of an invariant measure, supported on $B_{\lambda}$, by means of an approximating sequence of measures, different from the ones used by Brolin [8, p. 126], with the advantage that they are related to the measures which come from associated orthogonal polynomials. A related reference is [16].

Let $\Omega$ denote the set of all semi-infinite sequences of numbers from $\{-1,+1\}$. Then $\omega \in \Omega$ if and only if

Let

$$
\omega=\left(e_{1}, e_{2}, e_{3}, \ldots\right), \quad e_{i} \in\{-1,+1\} .
$$

$$
a=\lambda+\frac{1}{2}+\sqrt{\lambda+1 / 4} \text { and } I=[\lambda-\sqrt{a}, a] .
$$

Note that $a$ is the unique nonnegative real solution of $a=\lambda+\sqrt{a}$ and that $\lambda-\sqrt{a} \geqq 0$, where the inequality is strict when $\lambda>2$.

We define for $n \in\{0,1,2, \ldots\}$

$$
\begin{aligned}
& s_{0}(\omega, x)=x, \\
& s_{n}(\omega, x)=\lambda+e_{1} \sqrt{\left(\lambda+e_{2}\right.} \sqrt{\left(\lambda+\ldots+e_{n}\right.} \sqrt{(x) \ldots)) .}
\end{aligned}
$$

Then it is readily proved that $s_{n}: \Omega \times I \rightarrow I$.

We introduce a measure $\mu$ on $I$ by

$$
\mu E=\int_{E} \frac{d w}{\sqrt{w(2 \lambda-w)}}
$$

for all Lebesgue measurable subsets $E$ of $I$. Then $\mu$ is absolutely continuous with respect to Lebesgue measure on $I$ because $\int_{I} \frac{d w}{\sqrt{w(2 \lambda-w)}}<\infty$. Also, Lebesgue measure is absolutely continuous with respect to $\mu$ on $I$ because $\int_{E} d x=\int_{E} \sqrt{w(2 \lambda-w)} d \mu(\omega)$. The corresponding distance function is

$$
d(x, y)=|F(x)-F(y)| \text { for } \quad x, y \in I,
$$

where we define

$$
F(x)=\int_{0}^{x} \frac{d w}{\sqrt{w(2 \lambda-w)}} .
$$

Lemma 1. Let $\lambda \in[2, \infty), \varrho=\frac{1}{2} \cdot \sqrt{\frac{\lambda+\sqrt{a}}{\lambda^{2}-\lambda+\sqrt{a}}}$, and $n \in\{0,1,2, \ldots\}$. Then

$$
d\left(s_{n}(\omega, x), s_{n}(\omega, y)\right) \leqq \varrho^{n} d(x, y),
$$

where $\varrho \leqq 1 / 2$, the latter inequality being strict for $\lambda>2$. 
Proof. For $x<y$ and $e \in\{-1,+1\}$ we have by Cauchy's mean value theorem

$$
\begin{aligned}
\frac{d(\lambda+e \sqrt{x}, \lambda+e \sqrt{y})}{d(x, y)} & =\frac{|F(\lambda+e \sqrt{x})-F(\lambda+e \sqrt{y})|}{|F(x)-F(y)|} \\
& =\left|\frac{1}{2} \cdot \frac{e}{\sqrt{C}} \cdot \frac{F^{\prime}(\lambda+e \sqrt{C})}{F^{\prime}(C)}\right|=\frac{1}{2} \sqrt{\frac{2 \lambda-C}{\lambda^{2}-C}}
\end{aligned}
$$

for some $C \in(x, y)$. Since $0 \leqq \lambda-\sqrt{a} \leqq x<C<y \leqq a<2 \lambda \leqq \lambda^{2}$, the right-hand-side is bounded above by $\varrho$. This establishes the lemma for $n=1$ and induction completes the proof. Q.E.D.

Lemma 2. Let $\omega \in \Omega, x \in I$ and $\lambda \in[2, \infty)$. Then $s(\omega)=\operatorname{Lim}_{n \rightarrow \infty} s_{n}(x, \omega)$ exists, belongs to $I$, and is a constant independent of $x \in I$. For $\lambda \in(2, \infty), s: \Omega \rightarrow I$ is one-to-one.

Proof. We have from Lemma 1

$$
\begin{aligned}
d\left(s_{n+1}(x, \omega), s_{n}(x, \omega)\right) & =d\left(s_{n}\left(\lambda+e_{n+1} \sqrt{x} ; \omega\right), s_{n}(x, \omega)\right) \\
& \leqq \varrho^{n} d\left(\lambda+e_{n+1} \sqrt{x}, x\right) \leqq \varrho^{n} d(\lambda-\sqrt{a}, a),
\end{aligned}
$$

from which it follows that $\left\{s_{n}(x, \omega)\right\}_{n=1}^{\infty}$ is a Cauchy sequence. If

$$
\operatorname{Lim}_{n \rightarrow \infty} s_{n}(x, \omega)=s(\omega) \text { and } \operatorname{Lim}_{n \rightarrow \infty} s_{n}(y, \omega)=\tilde{s}(\omega),
$$

then

$$
d(s(\omega), \tilde{s}(\omega)) \leqq d\left(s(\omega), s_{n}(x, \omega)\right)+d\left(\tilde{s}(\omega), s_{n}(y, \omega)\right)+d\left(s_{n}(x, \omega), s_{n}(y, \omega)\right),
$$

which can be made arbitrarily small by choosing $n$ sufficiently large. The one-tooneness follows at once from the fact that $0 \notin I$ for $\lambda>2$. Q.E.D.

We now observe that the Julia set for $T_{\lambda} z=(z-\lambda)^{2}, \lambda \in[2, \infty)$ is precisely

$$
B_{\lambda}=\{s(\omega) \mid \omega \in \Omega\}
$$

This follows from the fact that the Julia set is the set of all limit points of all finite order preimages of any point in the plane, with at most two exceptions, [8]. The relationship between $B_{\lambda}$ and the collection of formal objects $\tilde{B}_{\lambda}$ is clear from this construction, and we will sometimes use the " $\lambda$-chain" notation

$$
s(\omega)=\lambda+e_{1} \sqrt{(} \lambda+e_{2} \sqrt{(} \lambda+e_{3} \sqrt{(\ldots)))} .
$$

The known properties of $B_{\lambda}$, given by Brolin [8] and summarized in the following theorem, are now straightforwardly deduced.

Theorem 1. For $\lambda \in[2, \infty), B_{\lambda}$ is perfect and totally invariant under $T_{\lambda}$, with

$$
T_{\lambda} s(\omega)=s(T \omega) \text { for all } \omega \in \Omega \text {, }
$$

where $T: \Omega \rightarrow \Omega$ is the left-shift operator. For $\lambda \in(2, \infty), B_{\lambda}$ is of Lebesgue measure zero. 
Proof. Since for any $\omega \in \Omega$ and $x \in I$ we have

$$
T_{\lambda}^{-1} s(\omega)=\left\{\operatorname{Lim}_{n \rightarrow \infty} s_{n}(\sigma, x) \mid \sigma \in T^{-1} \omega\right\}=s\left(T^{-1} \omega\right),
$$

and since $T^{-1} \Omega=\Omega$, it follows that both $T_{\lambda}^{-1} B_{\lambda}=B_{\lambda}$ and $T_{\lambda} s(\omega)=s(T \omega)$.

Every element of $B_{\lambda}$ is a limit point of other distinct elements of $B_{\lambda}$ because if $\sigma_{n} \in \Omega$ agrees with $\sigma \in \Omega$ in the first $n$ components then $\operatorname{Lim}_{n \rightarrow \infty} s\left(\sigma_{n}\right)=s(\sigma)$. (We can ensure $\left\{s\left(\sigma_{n}\right)\right\}$ contains infinitely many distinct elements even when $\lambda=2$ since for $\lambda=2, s: \Omega \rightarrow B_{\lambda}$ is at most two-to-one.) Similarly, $B_{\lambda}$ is compact because any infinite sequence in $\Omega$ contains a subsequence $\left\{\sigma_{n}\right\}$ and an element $\sigma$ such that $\sigma_{n}$ agrees with $\sigma$ in the first $n$ components. Hence $B_{\lambda}$ is perfect.

Since $B_{\lambda} \subset I$ we have $B_{\lambda} \subset T_{\lambda}^{-n} I=I_{n}$, where $I_{n}$ consists of $2^{n}$ intervals, each of which can be written $s_{n}(\omega, I)$ for some ${ }^{\prime} \omega \in \Omega$. Using Lemma 1 we readily calculate

$$
\mu\left(B_{\lambda}\right) \leqq \mu\left(I_{n}\right) \leqq 2^{n} \varrho^{n} \mu(I),
$$

whence when $\lambda>2$, so that $\varrho<1 / 2$, we have $\mu\left(B_{\lambda}\right)=0$. Q.E.D.

Using our alternative measure $\mu$ we obtain a new estimate for the Hausdorff dimension of $B_{\lambda}$.

Theorem 2. For $\lambda \in(2, \infty)$ the Hausdorff dimension of $B_{\lambda}$ is bounded above by the number

$$
\frac{\ln 2}{\ln \left(\frac{\sqrt{2 \lambda^{2}-2 \lambda+a}}{\sqrt{a}}\right)} .
$$

Proof. This follows the same lines as [8, Theorem 12.2] except that here we use the distance function $d(x, y)$ in place of $|x-y|$, these being equivalent metrics when $\lambda \in(2, \infty)$. Consider the sequence of coverings $\left\{I_{n}\right\}$ introduced above and write $I_{n}$ as a union of disjoint closed intervals, $I_{n}=\bigcup_{m=1}^{2^{n}} I_{n}^{m}$. Let

$$
H_{n}(\alpha)=\sum_{m=1}^{2^{n}}\left(\mu\left(I_{n}^{m}\right)\right)^{\alpha} \quad \text { for } \quad 0<\alpha<\infty .
$$

Then using Lemma 1 we readily calculate

$$
H_{n+1}(\alpha) \leqq\left(2 \varrho^{\alpha}\right) H_{n}(\alpha)
$$

from which it follows that $\operatorname{Lim}_{n \rightarrow \infty} H_{n}(\alpha)$ will be finite if $2 \varrho^{\alpha}<1$, which corresponds to $\alpha>\left(\ln \frac{1}{2}\right) / \ln \varrho$. This implies that $\left(\ln \frac{1}{2}\right) / \ln \varrho$ is an upper bound to the Hausdorff dimension of $B_{\lambda}$. Q.E.D.

Brolin has given the following upper bounds for the Hausdorff dimension of $B_{\lambda}$ :

(i) $\frac{\ln 2}{\ln 2(2 \hat{\lambda}-a)^{1 / 2}}$, valid for $\lambda>2+\sqrt{2}$,

(ii) $\frac{\ln 2}{\exp \left\{-60\left(\ln \left(\frac{(2 \lambda-a)^{1 / 2}}{5}\right)\right)^{2}\right\}+\ln 2}$, valid for $2<\lambda \leqq 6$. 
Our bound improves over both of these, where they apply. We note that for $\lambda=5$, Theorem 2 yields the upper bound 0.564 whilst (i) gives 0.636 . Thus our bound is good enough, at $\lambda=5$, to distinguish $B_{\lambda}$ from the classical ternary set of Cantor, whose Hausdorff dimension is $\ln 2 / \ln 3=0.631$, see [18].

We next give a construction, involving a particular sequence $\left\{\sigma_{n}(x)\right\}$ of approximating distributions, for an invariant distribution $\sigma(x)$ of $T_{\lambda}$, supported upon $B_{\lambda}$. Here $\sigma(x)$ is an example of the equilibrium distributions described by Brolin [3, Chap. III], and the $\sigma_{n}(x)$ 's are related to the orthogonal polynomials given in the next section.

Let $K_{n}=T_{\lambda}^{-n} \lambda$, which consists of the $2^{n}$ real points $\lambda \pm \sqrt{(\lambda} \pm \sqrt{(\lambda} \pm \ldots \pm \sqrt{(\lambda)} \ldots))$ where there are $n$ plus - or - minus signs. Let

$$
\sigma_{n}(x)=\frac{1}{2^{n}} \sum_{y \in K_{n}} \theta(x-y),
$$

where $\theta(x)=0$ when $x \leqq 0$ and $\theta(x)=1$ when $x>0$. Thus, $\sigma_{n}(x)$ equals the proportion of members of $K_{n}$ which are less than $x$.

It is straightforward to prove that $\left\{\sigma_{n}(x)\right\}$ converges uniformly to a continuous distribution $\sigma(x)$, for $x \in \mathbb{R}$. It is also straightforward to show, and in any case it follows from Brolin, that $\sigma(x)$ provides an invariant measure under $T_{\lambda}$, according to

$$
\int_{E} f(x) d \sigma(x)=\int_{T_{\lambda}^{-1}{ }^{1} E} f(T x) d \sigma(x)
$$

for all Borel measurable subsets $E$ of $\mathbb{R}$ and all measurable functions $f$. When $\lambda=2$, $T_{2} z=(z-2)^{2}$, and we have [23]

$$
d \sigma(x)=\left\{\begin{array}{lll}
0 & \text { for } \quad x \leqq 0 \\
\frac{1}{\pi} \frac{d x}{\sqrt{x(4-x)}} & \text { for } & 0<x<4 \\
0 & \text { for } & x>4
\end{array}\right.
$$

Let $F$ denote the set of all Borel measurable subsets of $B_{\lambda}$. Then $\left(B_{\lambda}, F, \sigma, T_{\lambda}\right)$ is a system as defined by Billingsley [6]. It is readily proved to be isomorphic to the system formed by the left-shift on $\Omega$ with the usual uniform measure. Consequently $\left(B_{\lambda}, F, \sigma, T_{\lambda}\right)$ is mixing with entropy $\ln 2$. The system is also isomorphic to the one formed by $z \rightarrow z^{2}$ on the unit circle in $\mathbb{C}$, with circular Lebesgue measure. This is one way to see the connection between the system which exists when $\lambda \geqq 2$ and that which exists when $\lambda=0$.

\subsection{Orthogonal Polynomials}

One way of characterizing the invariant measure $\sigma$ when $2 \leqq \lambda<\infty$ is by means of the associated set of monic orthogonal polynomials. We denote this set by $\left\{P_{n}(x)\right\}_{n=-1}^{\infty}$, where $P_{-1}(x) \equiv 0$. For $n \geqq 0, P_{n}(x)$ has degree $n$ and the coefficient of $x^{n}$ is unity. The polynomials obey

$$
\int_{I} P_{n}(x) P_{m}(x) d \sigma(x)=0 \quad \text { for } \quad n \neq m
$$


These polynomials provide an interesting generalization of the Tchebycheff polynomials $\left\{T_{n}(x)=\cos \left(n \cos ^{-1} x\right)\right\}$ to which, in view of the explicit formula for the measure given at the end of the last section, they must be related by

$$
P_{n}(x)=2 T_{n}\left(\frac{1}{2} x-1\right) \quad \text { when } \quad \lambda=2 .
$$

One would like to know how the invariance of the measure under $T_{\lambda}$ relates to the structure of the polynomials. Also, what can be said about the associated threeterm recurrence relations?

Let us introduce a second set of monic orthogonal polynomials $\left\{Q_{n}(x)\right\}_{n=-1}^{\infty}$, where $Q_{-1}(x) \equiv 0$. For $n \geqq 0, Q_{n}(x)$ has degree $n$ and the coefficient of $x^{n}$ is unity. They obey

$$
\int_{I} x Q_{n}(x) Q_{m}(x) d \sigma(x)=0 \quad \text { for } \quad n \neq m
$$

Then we have, for $n \geqq 0$ and $2 \leqq \lambda<\infty,[9,14,22]$

$$
Q_{n}(x)=\frac{1}{x}\left[P_{n+1}(x)-P_{n+1}(0) P_{n}(x) / P_{n}(0)\right] .
$$

We also define a set of polynomials $\left\{S_{n}(x)\right\}_{n=-1}^{\infty}$ by $S_{-1}(x) \equiv 0$ and

$$
\left.\begin{array}{rl}
S_{2 m}(x) & =P_{m}\left(x^{2}\right), \\
S_{2 m+1}(x) & =x Q_{m}\left(x^{2}\right),
\end{array}\right\} \text { for } m=0,1,2, \ldots .
$$

Theorem 3. For $\lambda \in[2, \infty)$,

$$
S_{n}(x-\lambda)=P_{n}(x)
$$

Proof. Clearly $S_{n}(x-\lambda)$ is a monic polynomial of degree $n$, when $n \geqq 0$. It remains only to prove that $\left\{S_{n}(x-\lambda)\right\}_{n=-1}^{\infty}$ is a set of polynomials orthogonal with respect to $\sigma$. Consider first for $n \neq m$

$$
\begin{aligned}
\int_{I} S_{2 n+1}(x-\lambda) S_{2 m+1}(x-\lambda) d \sigma(x) & =\int_{I}(x-\lambda)^{2} Q_{n}\left((x-\lambda)^{2}\right) Q_{m}\left((x-\lambda)^{2}\right) d \sigma(x) \\
& =\int_{I} Q_{n}(x) Q_{m}(x) x d \sigma(x)=0,
\end{aligned}
$$

where we have exploited the invariance of the measure $\sigma$ under $T_{\lambda}$. Next consider

$$
\begin{aligned}
\int_{I} S_{2 n}(x-\lambda) S_{2 m}(x-\lambda) d \sigma(x) & =\int_{I} P_{n}\left((x-\lambda)^{2}\right) P_{m}\left((x-\lambda)^{2}\right) d \sigma(x) \\
& =\int_{I} P_{n}(x) P_{m}(x) d \sigma(x)=0,
\end{aligned}
$$

where we have again used the invariance of the measure. Finally consider, for $m$ and $n$ not necessarily distinct,

$$
\int_{I} S_{2 n}(x-\lambda) S_{2 m+1}(x-\lambda) d \sigma(x)=\int_{I}(x-\lambda) P_{n}\left((x-\lambda)^{2}\right) Q_{m}\left((x-\lambda)^{2}\right) d \sigma(x) .
$$

This is zero because the integrand is antisymmetric about the midpoint $\lambda$ of I. Q.E.D.

Upon combining (1), (2), and (3) one finds

$$
P_{2 n}(x+\lambda)=P_{n}\left(x^{2}\right)
$$


and

$$
P_{2 n+1}(x+\lambda)=\frac{1}{x}\left[P_{n+1}\left(x^{2}\right)-\frac{P_{n+1}(0)}{P_{n}(0)} P_{n}\left(x^{2}\right)\right] .
$$

These two equations provide a bootstrap procedure for calculating the $P_{n}(x)$ 's, as indicated in the following scheme:

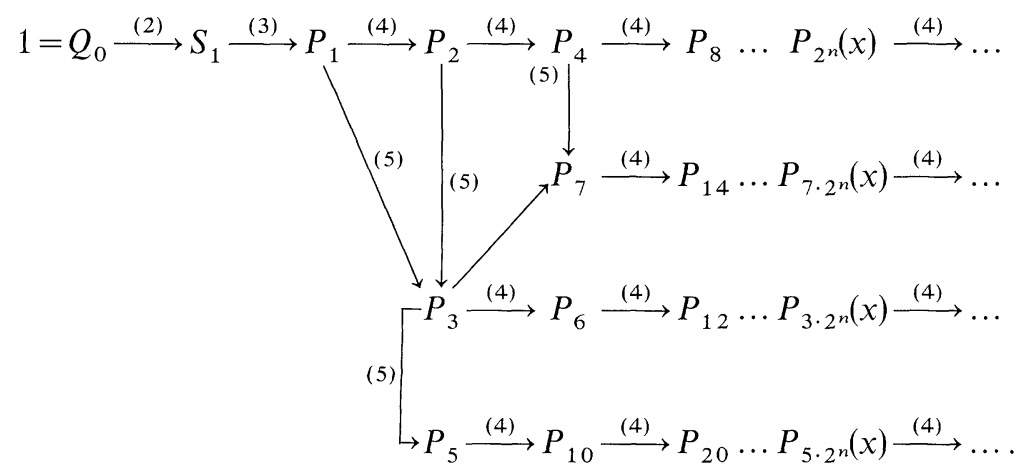

The numbers in parentheses indicate the equation to be used to travel along the arrows. Some examples of the resulting formulas are

$$
\begin{aligned}
P_{1}(x) & =x-\lambda, \\
P_{2^{n}}(x) & =\left(\ldots\left((x-\lambda)^{2}-\lambda\right)^{2} \ldots-\lambda\right)^{2}-\lambda, \quad n \geqq 1, \\
P_{3}(x) & =(x-\lambda)\left((x-\lambda)^{2}-\lambda-1\right), \\
P_{3 \cdot 2^{n}}(x) & =P_{2^{n}}(x)\left(P_{2^{n+1}}(x)-1\right), \quad m \geqq 0, \\
P_{5}(x) & =(x-\lambda)\left[(x-\lambda)^{4}-\frac{\left(2 \lambda^{2}+2 \lambda-1\right)}{\lambda-1}(x-\lambda)^{2}+\frac{\lambda^{3}-2 \lambda^{2}+2 \lambda+1}{\lambda-1}\right] .
\end{aligned}
$$

Notice that the set of zeros of $P_{2^{n}}(x)$ is precisely the set $K_{n}$ which was used in the construction $\sigma$. Similarly one can give expressions for all the zeros of $P_{3 \cdot 2^{n}}(x)$ and $P_{5 \cdot 2 n}(x)$.

We next consider the three-term recurrence relation satisfied by the polynomials.

Theorem 4. For $2 \leqq \lambda<\infty$ there exists a unique set of real numbers $\left\{a_{m}\right\}_{m=1}^{\infty}$ such that for $n \in\{1,2,3, \ldots\}$

$$
\begin{gathered}
P_{n+1}(x)=(x-\lambda) P_{n}(x)-a_{n}^{2} P_{n-1}(x), \\
\lambda-a_{2 n+1}^{2}-a_{2 n}^{2}=0,
\end{gathered}
$$

and

$$
a_{n}^{2}=a_{2 n}^{2} a_{2 n-1}^{2} .
$$

Proof. Since the measure $\sigma(x)$ is symmetric about $x=\lambda$ it follows at once from the theory of orthogonal polynomials that there exists a unique set of real numbers $\left\{a_{m}\right\}_{m=1}^{\infty}$ such that the three-term recurrence formula (6) holds. To prove (7) and (8) 
it is convenient to work in terms of the shifted polynomials $S_{n}(x)=P_{n}(x+\lambda)$ : we consider

$$
S_{2 n+2}(x)=x S_{2 n+1}(x)-a_{2 n+1}^{2} S_{2 n}(x) .
$$

Eliminating $S_{2 n+1}(x)$ from this expression by using the recurrence relation we obtain

$$
S_{2 n+2}(x)=\left(x^{2}-a_{2 n+1}^{2}\right) S_{2 n}(x)-x a_{2 n}^{2} S_{2 n-1}(x) .
$$

Herein we reexpress $S_{2 n-1}(x)$ in terms of $S_{2 n}(x)$ and $S_{2 n-2}(x)$, again with the aid of the recurrence equation, to obtain

$$
S_{2 n+2}(x)=\left(x^{2}-a_{2 n+1}^{2}-a_{2 n}^{2}\right) S_{2 n}(x)-a_{2 n}^{2} a_{2 n-1}^{2} S_{2 n-2}(x) .
$$

We now set $x^{\prime}=x^{2}-\lambda$ and use the fact that

$$
S_{2 m}(x)=S_{m}\left(x^{2}-\lambda\right) \quad \text { for } \quad m \in\{0,1,2, \ldots\} ;
$$

which yields

$$
S_{n+1}\left(x^{\prime}\right)=\left(x^{\prime}+\lambda-a_{2 n+1}^{2}-a_{2 n}^{2}\right) S_{n}\left(x^{\prime}\right)-a_{2 n}^{2} a_{2 n-1}^{2} S_{n-1}\left(x^{\prime}\right) .
$$

Equations (7) and (8) follow at once upon comparing with the recurrence relation $S_{n+1}(x)=x S_{n}(x)-a_{n}^{2} S_{n-1}(x)$. Q.E.D.

With the aid of (7) and (8) we readily calculate

$$
\begin{gathered}
a_{1}^{2}=\lambda, \quad a_{2}^{2}=1, \quad a_{3}^{2}=\lambda-1, \\
a_{4}^{2}=\frac{1}{\lambda-1}, \quad a_{5}^{2}=\frac{\lambda^{2}-\lambda-1}{\lambda-1}, \quad a_{6}^{2}=\frac{\lambda^{2}-2 \lambda+1}{\lambda^{2}-\lambda-1} .
\end{gathered}
$$

We also obtain the following continued fractions representation for $a_{n}$ : for $n \geqq 2$

$$
a_{2 n}^{2}=\frac{a_{n}^{2} \mid}{\mid \lambda}-\frac{a_{n-1}^{2} \mid}{\mid \lambda} \ldots-\frac{a_{2}^{2} \mid}{\mid \lambda-1},
$$

and

$$
a_{2 n+1}^{2}=\lambda-\frac{a_{n}^{2} \mid}{\mid \lambda}-\frac{a_{n-1}^{2} \mid}{\mid \lambda} \ldots-\frac{a_{2}^{2} \mid}{\mid \lambda-1}
$$

In particular, when $\lambda=2$ we have $a_{n}^{2}=1$ for $n>2$ and $a_{1}^{2}=2$, and (6) becomes exactly the three-term recursion relation for the Tchebycheff polynomials $\left\{T_{n}\left(\frac{1}{2} x-1\right)\right\}_{n=-1}^{\infty}$. From this it follows that the zeros of $T_{2^{n}}\left(\frac{1}{2} x-1\right)$ are precisely the set of numbers

$$
\begin{gathered}
2 \pm \sqrt{(2} \pm \sqrt{(2} \pm \ldots \pm \sqrt{2}) \ldots) \\
\qquad n \text { times }
\end{gathered}
$$

The densification of the latter set of numbers on $[0,4]$ can thus be seen as an example of Blumenthal's theorem [7] on the distribution of zeros of orthogonal polynomials upon the support of the measure.

Further information, which relates in particular to the sequence of approximating measures $\left\{\sigma_{n}(x)\right\}$ given in Sect. 2.1, is obtained by examining the 
polynomials of the second kind, $\left\{P_{n}^{1}(x)\right\}_{n=0}^{\infty}$, which are defined by

$$
P_{n}^{1}(x)=\int_{I} \frac{P_{n+1}(x)-P_{n+1}(y)}{x-y} d \sigma(y) \text { for } n \in\{-1,0,1,2 \ldots\} .
$$

Theorem 5. For all $n \in\{0,1,2, \ldots\}$,

$$
P_{2 n+1}^{1}(x)=(x-\lambda) P_{n}^{1}\left((x-\lambda)^{2}\right)
$$

and

$$
P_{2 n}(x)=\frac{1}{(x-\lambda)}\left[P_{2 n+1}^{1}(x)-\frac{P_{2 n+2}(\lambda)}{P_{2 n}(\lambda)} P_{2 n-1}^{1}(x)\right] .
$$

Proof. From (9) one has

$$
P_{n}^{1}\left((x-\lambda)^{2}\right)=\int_{I} \frac{P_{n+1}\left((x-\lambda)^{2}\right)-P_{n+1}\left((y-\lambda)^{2}\right)}{(x-\lambda)^{2}-(y-\lambda)^{2}} d \sigma(y),
$$

where the invariance of the measure under $T_{\lambda}$ has been exploited. We now split up the denominator and use (4), which yields

$$
\begin{aligned}
P_{n}^{1}\left((x-\lambda)^{2}\right)= & \frac{1}{2(x-\lambda)}\left[\int_{I} \frac{P_{2 n+2}(x)-P_{2 n+2}(y)}{(x-\lambda)-(y-\lambda)} d \sigma(y)\right. \\
& \left.+\int_{I} \frac{P_{2 n+2}(x)-P_{2 n+2}(y)}{(x-\lambda)+(y-\lambda)} d \sigma(y)\right] .
\end{aligned}
$$

In the second integral here we make the change of variable $y-\lambda \rightarrow-(y-\lambda)$, use the symmetry of the measure and $I$ about $\lambda$, and again exploit (4), to provide

$$
P_{n}^{1}\left((x-\lambda)^{2}\right)=\frac{1}{(x-\lambda)} \int_{I} \frac{P_{2 n+2}(x)-P_{2 n+2}(y)}{(x-y)} d \sigma(y) .
$$

From this (10) is immediate.

Equation (9) also implies the recursion relation

$$
P_{n+1}^{1}(x)=(x-\lambda) P_{n}^{1}(x)-a_{n+1}^{2} P_{n-1}^{1}(x) \text { for } n \in\{0,1,2, \ldots\},
$$

with $P_{-1}^{1}(x)=0$, and $P_{0}^{1}(x)=1$. This implies (11) when $a_{n+1}^{2}$ is eliminated with the aid of (6) wherein $\lambda=x$. Q.E.D.

From (10) it is apparent, in contrast to the previous case, that the odd polynomials of the second kind are easily calculated from the even ones. Some examples of the polynomials are

$$
\begin{aligned}
P_{0}^{1}(x) & =1 \\
P_{1}^{1}(x) & =x-\lambda=P_{1}(x), \\
P_{3}^{1}(x) & =(x-\lambda)\left((x-\lambda)^{2}-\lambda\right)=P_{1}(x) P_{2}(x)=\frac{1}{4} \frac{d}{d x} P_{4}(x), \\
P_{2^{n-1}}^{1}(x) & =\prod_{k=0}^{n-1} P_{2^{k}}(x)=\frac{1}{2^{n}} \frac{d}{d x} P_{2^{n}}(x), \quad n \geqq 1 \\
P_{3 \cdot 2^{n-1}}^{1}(x) & =\left(\prod_{k=0}^{n-1} P_{2^{k}}(x)\right)\left(P_{2^{n+1}}(x)+\lambda-1\right) .
\end{aligned}
$$


Now consider the moment functions

$$
G_{K}(x)=\int_{I} \frac{d \sigma_{K}(y)}{x-y}
$$

and

$$
G(x)=\int_{I} \frac{d \sigma(y)}{x-y} .
$$

From the theory of Padé approximants [2], one has that the $[n-1 / n](x)$ approximant to $G(x)$ is

$$
[n-1 / n](x)=\frac{P_{n-1}^{1}(x)}{P_{n}(x)}, \text { for } n \in\{1,2, \ldots\} .
$$

Using (4) and the fact that $S_{2 m}(x)=S_{m}\left(x^{2}-\lambda\right)$ we discover the remarkable result

$$
[2 n-1 / 2 n](x)=(x-\lambda)[n-1 / n]\left((x-\lambda)^{2}\right) \text { for } n=1,2,3, \ldots .
$$

Also, for $n=2^{k}$ where $k \in\{0,1,2, \ldots\}$, we find

$$
\left[2^{k}-1 / 2^{k}\right](x)=\frac{1}{2^{k}} \frac{d}{d x} \ln P_{2^{k}}(x)=\int_{I} \frac{d \sigma_{K}(x)}{x-y},
$$

which makes contact with the sequence of approximating measures $\left\{\sigma_{n}(x)\right\}$.

Finally, we note that since $\left(B_{\lambda}, F, \sigma, T_{\lambda}\right)$ is a mixing system, so is $\left(B_{\lambda}, F, \sigma, T_{\lambda}^{n}\right)$ for $n \in\{1,2,3, \ldots\}$. Hence $P_{2^{n}}(x)+\lambda$ provides a mixing transformation on $B_{\lambda}$, with respect to $\sigma$. Shifting $B_{\lambda}$ to the left by subtracting $\lambda$, and correspondingly adjusting the measure, this shows that each of the polynomials $P_{2^{n}}(x+\lambda)$ provides a mixing transformation upon the shifted system. This extends a result of Adler and Rivlin [1], and is itself a special case of a wide reaching theorem [5].

\section{The Cases $-1 / 4 \leqq \lambda \leqq 2$ and $|\lambda| \leqq 1 / 4$ with $\lambda \in \mathbb{C}$}

In this section, the Julia set $B_{\lambda}$ is connected, and a central role is played by the Böttcher equation, see [12], for $T_{\lambda}$ at $\infty$

$$
T_{0} \circ H_{\lambda}=H_{\lambda} \circ T_{\lambda}, \quad H_{\lambda}(z)=z+O(1) \text { at } \infty .
$$

We actually use the equivalent equation in terms of inverses, where $F_{\lambda}=H_{\lambda}^{-1}$,

$$
F_{\lambda}(z)=\lambda+\sqrt{F_{\lambda}\left(z^{2}\right)}, \quad F_{\lambda}(z)=z+O(1) \text { at } \infty .
$$

We let $\mathbb{C}$ be the complex plane, $\hat{\mathbb{C}}=\mathbb{C} \cup\{\infty\}$ and $D_{0}=\{z \in \hat{\mathbb{C}}|| z \mid>1\}$. Then we shall see that $F_{\lambda}$ maps $D_{0}$ conformally onto a region bounded by $B_{\lambda}$, and by means of $F_{\lambda}$ we can relate $\lambda$-chains to $B_{\lambda}$.

\subsection{Two Constructions for $B_{\lambda}$}

We present two constructions for $B_{\lambda}$; one from the "outside", and one from the "inside", when $-\frac{1}{4} \leqq \lambda \leqq 2$. The first is not new in principle: it involves the 
formation of an increasing sequence of domains, successive inverse images under $T_{\lambda}$ of a neighborhood of $\infty$, as suggested by Fatou [12] and by Julia [19]. We include it both for completeness and for comparison with the second method.

The second construction, from the "inside", provides a decreasing sequence of domains and a corresponding sequence of functions, from $D_{0}$ to the domains, converging uniformly to $F_{\lambda}(z)$ on compact subsets of $D_{0}$. Of interest are the complements of the domains, which form an increasing sequence of trees, with fractal-like structure [20] and two-dimensional measure zero, which serve to describe $B_{\lambda}$ from the interior. This construction turns out to be important: we have recently proved [4] that this sequence of trees converges to $B_{\lambda}$ itself, for infinitely many values of $\lambda \in(0,2)$.

For $\lambda \geqq-\frac{1}{4}$ we define

$$
a=\sqrt{\lambda+1 / 4}+\lambda+1 / 2, \text { and } b=\sqrt{|\lambda|+1 / 4}+|\lambda|+1 / 2 .
$$

Then $a$ is the unique positive real numbers which obeys $a=\lambda+\sqrt{a}$. Notice that $a \leqq b$, and $\lambda-\sqrt{a} \leqq 0$, for $-\frac{1}{4} \leqq \lambda \leqq 2$.

The following Lemma will allow us to make a concrete iterative solution of the Böttcher equation (1b). This construction is important later on in our discussion of Hölder continuity.

Lemma 3. For $-\frac{1}{4} \leqq \lambda<2$, we may start from $f_{0}(z)=\lambda+\sqrt{b} z$ and iteratively define analytic functions $f_{n}: D_{0} \rightarrow \hat{\mathbb{C}}$ for $n \in\{1,2,3, \ldots\}$ by

$$
f_{n}(z)=\lambda+\sqrt{f_{n-1}\left(z^{2}\right)}=b^{2-(n+1)} z+\lambda+O\left(\frac{1}{z}\right),
$$

and they will satisfy

$$
\hat{\mathbb{C}} \backslash[\lambda-\sqrt{a}, a] \supset f_{n}\left(D_{0}\right) \supset f_{n-1}\left(D_{0}\right) .
$$

Sketch of Proof. The lemma can be easily proved by induction. The exclusion of the interval $[\lambda-\sqrt{a}, a]$, which contains zero, follows from the facts that $a \leqq b$ and $T_{\lambda}$ maps the excluded interval into itself. Thus, $f_{n}$ is well defined because $0 \notin f_{n-1}\left(D_{0}\right)$. That $f_{1}\left(D_{0}\right) \supset f_{0}\left(D_{0}\right)$ is a simple calculation using the definition of $b$, and upon iteration we obtain the monotonicity of the images.

Theorem 6. Let $-\frac{1}{4} \leqq \lambda \leqq 2$. The sequence $\left\{f_{n}\right\}$ of Lemma 3 converges uniformly on compact subsets of $D_{0}$ to a function $F_{\lambda}$ which obeys the inverse Böttcher equation (1b).

Remark. We denote the boundary of $F_{\lambda}\left(D_{0}\right)$ by $B_{\lambda}$. The theorem says $T_{\lambda}^{-1} B_{\lambda}=B_{\lambda}$. Then $B_{\lambda}$ turns out to be the Julia set for $T_{\lambda}$, see [12] for example.

Proof. The theorem follows at once from Lemma 3 and Caratheodory's theorem on domain convergence, see Goluzin [15, p. 53]. Q.E.D.

We can now set up a correspondence beţween $\lambda$-chains and points on $B_{\lambda}$. Corresponding to $\omega=\left(e_{1}, e_{2}, e_{3}, \ldots\right) \in \Omega$ we define $S_{0}(\omega, z)=z$ and, for $n \in\{1,2,3, \ldots\}$,

$$
S_{n}(\omega, z)=\lambda+e_{1} V\left(\lambda+e_{2} V\left(\lambda+\ldots+e_{n-1} V\left(\lambda+e_{n} \sqrt{z}\right) \ldots\right)\right) .
$$


The value of the square root $\sqrt{w}$ for $w \in \mathbb{C}$ is fixed by writing $w=\Gamma e^{i \theta}$ with $0 \leqq \theta<2 \pi$, and then

$$
\sqrt{z}=\sqrt{\Gamma e^{i \theta / 2}}, \text { and }-\sqrt{z}=-\sqrt{\Gamma e^{i \theta / 2}}=\sqrt{\Gamma e^{i(\theta+2 \pi) / 2}} .
$$

We will say that we have a positive axis branch cut. Thus $S_{n}(\omega, z)$ is clearly a well defined function of its arguments.

Theorem 7. Let $-\frac{1}{4} \leqq \lambda \leqq 2$ and $0 \leqq \theta<2 \pi$. Write $\theta=2 \pi \sum_{j=1}^{\infty} d_{j} / 2^{j}$, where $d_{j} \in\{0,1\}$. Let $e_{j}=+1$ if $d_{j}=0, e_{j}=-1$ if $d_{j}=1$, and $\omega=\left(e_{1}, e_{2}, e_{3}, \ldots\right)$. Then

i) $\operatorname{Lim}_{n \rightarrow \infty} F_{\lambda}^{-1}\left(S_{n}(\omega, z)\right)=e^{i \theta}$ and

(ii) $\operatorname{Lim}_{n \rightarrow \infty} S_{n}(\omega, z)$ exists for almost all $\theta$, independent of $z \in F_{\lambda}\left(D_{0}\right)$.

Remark. We define the $\lambda$-chain $S(\omega)$ to be the limit of $S_{n}(\omega, z)$ when it exists.

Proof. Let $z=F_{\lambda}\left(\Gamma e^{i \alpha}\right), 0 \leqq \alpha<2 \pi, \Gamma>1$. From symmetry observe that $F_{\lambda}$ maps each of the upper half plane, the lower half plane, the positive axis and the negative axis into itself. Hence when we use the Böttcher equation (1b) we can choose the appropriate branches of the successive square roots and find

From this (i) follows.

$$
F_{\lambda}^{-1}\left(S_{n}(\omega, z)\right)=\Gamma^{2-n} e^{i \alpha / 2^{n}} e^{2 \pi i} \sum_{j=1}^{n} d_{j} / 2^{j}
$$

It is easy to show that in (i) the limit $e^{i \theta}$ on the unit circle is approached nontangentially. Since $F_{\lambda}(z)-z$ is regular and bounded on $D_{0}$, it has nontangential limits almost everywhere on the unit circle, Goluzin [15, p. 343], and we find that $\left\{S_{n}(\omega, z)\right\}$ has a unique limit point for almost every $\theta$. Q.E.D.

The power series coefficients of the unique solution of the Böttcher equation (1b) can be calculated recursively. For example, after several iterations we obtain

$$
F_{\lambda}(z)=z+\lambda+\frac{\lambda}{2 z}+\frac{\lambda(2-\lambda)}{8 z^{2}}-\frac{\lambda^{2}(2-\lambda)}{16 z^{5}}+O\left(\frac{1}{z^{7}}\right)
$$

When $\lambda=2$ this reduces to $F_{2}(z)=z+2+\frac{1}{z}$.

In order to obtain more detailed information about $B_{\lambda}$ it is useful to construct a second sequence of functions $\left\{f_{n}^{*}(z)\right\}$ which is convergent to $F_{\lambda}(z)$. Whereas the sequence $\left\{f_{n}(z)\right\}$ provides a sequence of images which increase to $F_{\lambda}\left(D_{0}\right)$ (whose boundary is $\left.B_{\lambda}\right)$, the sequence $\left\{f_{n}^{*}(z)\right\}$ yields decreasing images, and we get convergence to $B_{\lambda}$ from the "inside". The proof is similar to that of Lemma 3. Lemma 4. For $-\frac{1}{4} \leqq \lambda \leqq 2$ we may start with $f_{0}^{*}(z)=\lambda+\sqrt{a / 4}\left(z+\frac{1}{z}\right)$ and iteratively
define analytic functions $f_{n}^{*}: D_{0} \rightarrow \hat{\mathbb{C}}$ for $n \in\{1,2,3, \ldots\}$ by

$$
f_{n}^{*}(z)=\lambda+\sqrt{f_{n-1}^{*}\left(z^{2}\right)}=(a / 4)^{2-(n+1)} z+\lambda+O\left(\frac{1}{z}\right)
$$

and they will satisfy

$$
f_{n}^{*}\left(D_{0}\right) \subset f_{n-1}^{*}\left(D_{0}\right) \subset \hat{\mathbb{C}} \backslash[\lambda-\sqrt{a}, a]
$$




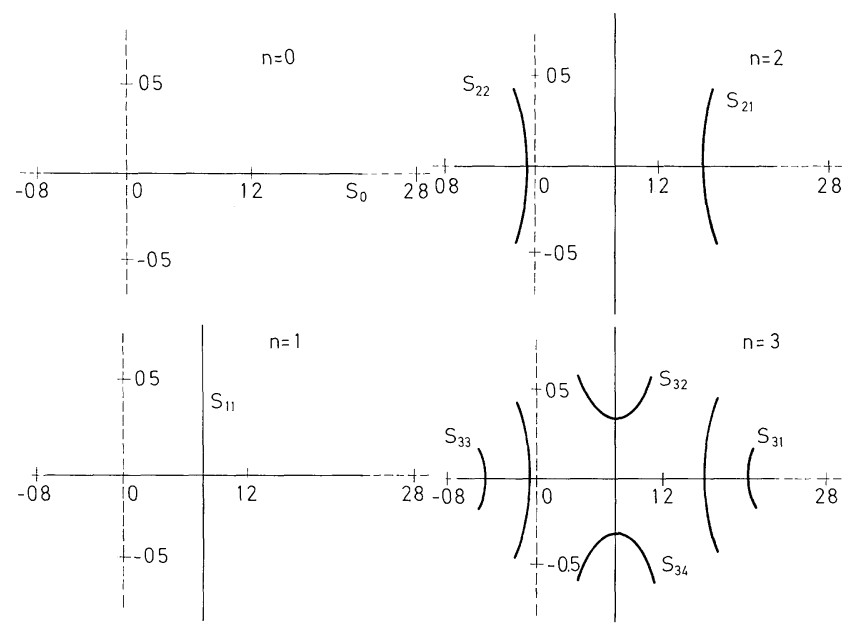

Fig. 1. The boundaries $\Gamma_{n}$ for $n=0,1,2$, and 3, are drawn unbroken; the dots lie upon the coordinate axes. In general, $\Gamma_{n+1}$ contains $2^{n}$ new arcs not in $\Gamma_{n}$, and $\Gamma_{0} \subset \Gamma_{1} \subset \Gamma_{2} \subset \Gamma_{3} \subset \ldots$

It turns out to be very useful to give a fairly complete characterization of the sets $\Gamma_{n}=f_{n}^{*}\left(\partial D_{0}\right)$. Let $S_{0}=[\lambda-\sqrt{a}, a]=\Gamma_{0}$. Then the image of $S_{0}$ under the principal branch of $\lambda+\sqrt{z}$ is $[\lambda, a] \cup[$ the closed line segment from $\lambda$ to $\lambda+i \sqrt{\sqrt{a}-\lambda]}$. Since $f_{1}^{*}(z)-\lambda$ is an odd function of $z$ we can reflect the latter set about $\lambda$ to obtain the rest of $\Gamma_{1}$. In this way we find

$$
\Gamma_{1}=S_{0} \cup S_{11} \supset \Gamma_{0}
$$

where $S_{11}$ is the closed line segment connecting $\lambda \pm \sqrt{\sqrt{a}-\lambda}$. It will be found helpful here to look at Fig. 1. Here $\Gamma_{2}$ is the image of $\Gamma_{1}$ under $\lambda \pm \sqrt{z}$. Then $S_{0}$ will generate $S_{0} \cup S_{11}$, but $S_{11}$ will produce two new analytic arcs denoted by $S_{21}$ and $S_{22}$. In general $\Gamma_{n}$ will contain $2^{n-1}$ new arcs not in $\Gamma_{n-1}$ :

$$
\Gamma_{n}=S_{0} \cup\left\{\bigcup_{j=1}^{n}\left\{\left(\bigcup_{k=1}^{2 j-1} S_{j k}\right)\right\}\right\}
$$

where each $S_{j k}$ is an analytic arc. The endpoints of $S_{0}$ are $\lambda \pm \sqrt{a}=F_{\lambda}( \pm 1)$, and by repeatedly using the Böttcher equation $(1 b)$ and the definition of $f_{n}^{*}$ we find that the endpoints of the arcs composing $\Gamma_{n}$ are $F_{\lambda}\left(e^{j \pi i / 2^{n}}\right)$ for all $j \in\left\{1,2, \ldots, 2^{n+1}\right\}$. Equivalently, the endpoints of $\Gamma_{n}$ are all numbers expressible in the form

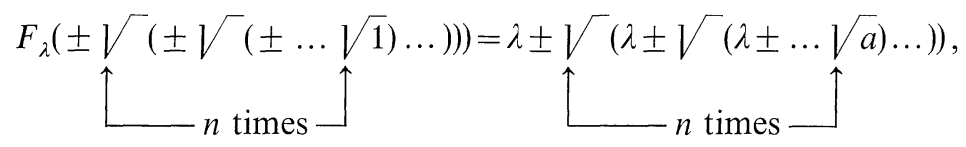

where \pm indicates that both branches of $\sqrt{ }$ can be chosen.

We can replace the $f_{n}$ of Theorem 6 by $f_{n}^{*}$ to obtain the following result.

Theorem 8. Let $-\frac{1}{4} \leqq \lambda \leqq 2$. Then the sequence of functions $\left\{f_{n}^{*}\right\}$ provided by Lemma 4 converges uniformly to $F_{\lambda}$ on compact subsets of $D_{0}$. 


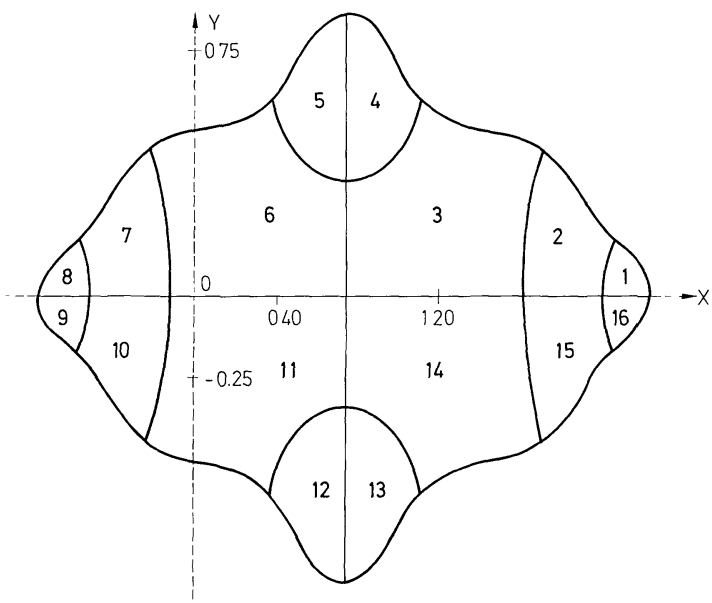

Fig. 2. The boundaries of $f_{3}\left(D_{0}\right)$ and $f_{3}^{*}\left(D_{0}\right)$ are superimposed, and sixteen components are thereby defined. The closure of the component labeled $j$ contains the part of $B_{\lambda}$ which corresponds to $\left\{e^{\imath \theta} \mid(j-1) \pi / 16 \leqq 0 \leqq j \pi / 16\right\}$

The proof is like that of Theorem 6. The uniqueness of the solution to the Böttcher equation ensures that the limit is $F_{\lambda}$.

If $K$ is a compact subset of $D_{0}$, then the sequence of sets $\left\{f_{n}(K)\right\}_{n=0}^{\infty}$ and $\left\{f_{n}^{*}(K)\right\}_{n=0}^{\infty}$ increase and decrease respectively to $F_{\lambda}(K)$. Similarly $\left\{f_{n}\left(D_{0}\right)\right\}_{n=1}^{\infty}$ increases to $F_{\lambda}\left(D_{0}\right)=D_{\lambda}$. However, the decreasing sequence $\left\{f_{n}^{*}\left(D_{0}\right)\right\}_{n=0}^{\infty}$ does not converge to $D_{\lambda}$. This sequence illustrates a peculiar property of domain convergence. For example, we will show in the next section that when $0 \leqq \lambda \leqq 1 / 4, D_{\lambda}^{c}$ contains the disk of radius $1 / 4$ about 0 . In this case the area of $D_{\lambda}^{c}$ is strictly positive, yet the area of $\Gamma_{n}$ is zero for all $n$.

The behavior of these image sets can be better understood by considering the endpoints of the analytic arcs in $\Gamma_{n}$. The set $\left\{\bigcup_{n=1}^{\infty} e^{j \pi i / 2^{n}} \mid j=1,2, \ldots, 2^{n+1}\right\}$ is dense in the unit circle $\partial D_{0}=B_{0}$, and its image under $F_{\lambda}$ is dense in $B_{\lambda}$. The latter image is precisely the set of endpoints of all of the analytic arcs in all of the $\Gamma_{n}$ 's. Thus, consistently with the general theory of domain convergence, we find $F_{\lambda}\left(D_{0}\right)$ is one component of the interior of $\operatorname{Lim}_{n \rightarrow \infty} f_{n}^{*}\left(D_{0}\right)$.

For $0<\lambda<2$ the numbers $\lambda \pm \sqrt{a}$ are on the boundary of both $f_{0}\left(D_{0}\right)$ and $f_{0}^{*}\left(D_{0}\right)$. Hence

$$
f_{n}(z)=f_{n}^{*}(z)=F_{\lambda}(z) \text { for } \quad z=e^{j \pi i / 2^{n}}
$$

for all $j \in\left\{1,2, \ldots, 2^{n+1}\right\}$ and $n \in\{0,1,2, \ldots\}$. If we superimpose the boundaries of $f_{n}\left(D_{0}\right)$ and $f_{n}^{*}\left(D_{0}\right)$ then we separate the complex plane into many components. The portion of $B_{\lambda}$ corresponding to $\left\{e^{i \theta} \mid(j-1) \pi / 2^{n} \leqq \theta \leqq j \pi / 2^{n}\right\}$ must lie in the closure of the $j^{\text {th }}$ component counted counter-clockwise, starting from the one in the first quadrant which has $a$ on its boundary. As an example we illustrate the case $n=3$ in Fig. 2 where the sixteen components are labeled. 
3.2. The Relation Between $B_{\lambda}$ and $\tilde{B}_{\lambda}$ when $|\lambda|<1 / 4$ with $\lambda \in \mathbb{C}$

First we restrict attention to $0 \leqq \lambda \leqq 0.2$ and make explicit calculations with $\lambda$-chains which show that $S(\omega)$ is always well-defined and that $F_{\lambda}$ can be extended continuously to the unit circle. Next we make analytic extensions to $\lambda$ in the set $L=\{\lambda \in \mathbb{C}|| \lambda \mid<1 / 4\}$ and show that the mappings $G_{\lambda}(\theta)=F_{\lambda}\left(e^{i \theta}\right)$ are uniformly Hölder continuous for $\lambda$ in any compact subset of $L$.

We introduce the notation

$$
S(+, z)=\lambda+\sqrt{z} \text { and } S(-, z)=\lambda-\sqrt{z},
$$

where the positive axis branch cut is used. Then we have the following two simple computational Lemmas whose proofs we omit.

Lemma 5. Let $\lambda \in \mathbb{R}$ and $z \in \mathbb{C}$ with $z \ngtr 0$. Then $S(+, z)=\overline{S(-, \bar{z})}$.

For $\lambda \in L$ and $d \geqq b$ let

$$
C=-|\lambda|+\frac{1}{2}+\sqrt{-|\lambda|+1 / 4}
$$

and

$$
R_{d}=\{z \in \mathbb{C}|C \leqq| z \mid \leqq d\} .
$$

Lemma 6. Let $\lambda \in L$ and $d \geqq b$. Then both $S(+, z)$ and $S(-, z)$ map $R_{d}$ into itself.

Let $\mu, \sigma$, and $\xi$ in $\Omega$ be defined by

$$
\begin{aligned}
& \mu=(-1,-1,-1,-1, \ldots), \\
& \sigma=(+1,-1,-1,-1, \ldots),
\end{aligned}
$$

and

$$
\xi=(-1,+1,+1,+1, \ldots) .
$$

Lemma 7. For $0<\lambda \leqq 2, S(\sigma)$ and $S(\xi)$ both exist and $S(\sigma)=S(\xi)=S(-, a)$.

Proof. We claim that $S(-, z)$ is a contraction mapping towards $a$ on $\{z \mid \operatorname{Im} z<0\}$ and that $S(+, z)$ is a contraction mapping towards $a$ on $\{z \mid \operatorname{Im} z>0\}$. By Lemma 5 these claims are equivalent, so we prove only the latter. For $\operatorname{Im} z>0$ we have

$$
\begin{aligned}
\left|\frac{S(+, z)-a}{z-a}\right| & =\left|\frac{\lambda+\sqrt{z}-(\lambda+\sqrt{a})}{z-a}\right|=\left|\frac{\sqrt{z}-\sqrt{a}}{z-a}\right| \\
& =\left|\frac{1}{\sqrt{z}+\sqrt{a}}\right| \leqq \frac{1}{\operatorname{Re}(\sqrt{z}+\sqrt{a})} \\
& \leqq \frac{1}{\sqrt{a}}<1
\end{aligned}
$$

where we have used the fact that $\operatorname{Re} \sqrt{z}>0$. This proves our claim. It now follows that the sequence $\left\{S_{n}(\mu, z)\right\}_{n=1}^{\infty}$ approaches a through the fourth quadrant, which, in turn implies that $\left\{\sqrt{S_{n}(\mu, z)}\right\}_{n=1}^{\infty}$ converges to $-\sqrt{a}$. Hence $\left\{S_{n}(\sigma, z)\right\}_{n=1}^{\infty}$ converges to $\lambda-\sqrt{a}$. Q.E.D. 
Lemma 8. Let $0<\lambda<0.2, d \geqq a, n \in\{1,2, \ldots\}$, and $\omega \in \Omega$. Then for any $z_{1}, z_{2} \in R_{d}$,

$$
\left|S_{n}\left(\omega, z_{1}\right)-S_{n}\left(\omega, z_{2}\right)\right| \leqq 2 d r^{n-3},
$$

where $r=0.87$.

Proof. First note that for any $w_{1}, w_{2} \in R_{d}$ we have

$$
\begin{aligned}
\left|\arg \sqrt{w_{1}}-\arg \sqrt{w_{2}}\right| & =\left|\arg \left(-\sqrt{w_{1}}\right)-\arg \left(-\sqrt{w_{2}}\right)\right| \\
& =1 / 2\left|\arg w_{1}-\arg w_{2}\right| .
\end{aligned}
$$

Also, for any $w \in R_{d}, \sqrt{w}$ lies in the upper half plane and

$$
\begin{aligned}
0 & \leqq \arg \sqrt{w}-\arg (\sqrt{w}+\lambda) \\
& \leqq \sin ^{-1}(\lambda / \sqrt{|w|}) \leqq \sin ^{-1}(0.2 / \sqrt{0.5})<0.3
\end{aligned}
$$

Here we have used the fact that when $0<\lambda<0.2$, then $C \geqq 0.5$ so that for all $w \in R_{d}$ we have $|w|>0.5$. Then we can show

$$
\left|\arg S\left( \pm, w_{1}\right)-\arg S\left( \pm, w_{2}\right)\right| \leqq 1 / 2\left|\arg w_{1}-\arg w_{2}\right|+0.3
$$

Lemma 6 allows us to iterate the above result starting from $z_{1}, z_{2} \in R_{d}$. We find

$$
\left|\arg S_{m}\left(\tilde{\omega}, z_{1}\right)-\arg S_{m}\left(\tilde{\omega}, z_{2}\right)\right| \leqq \beta=1.25<\frac{\pi}{2}
$$

for all $m \geqq 3$, for any $\tilde{\omega} \in \Omega$.

Again let us suppose $w_{1}, w_{2} \in R_{d}$, but now with $\left|\arg w_{1}-\arg w_{2}\right| \leqq \beta$. Then

$$
\begin{aligned}
\left|\frac{S\left( \pm, w_{1}\right)-S\left( \pm, w_{2}\right)}{w_{1}-w_{2}}\right| & =\left|\frac{\sqrt{w_{1}}-\sqrt{w_{2}}}{w_{1}+w_{2}}\right|=\frac{1}{\sqrt{w_{1}}+\sqrt{w_{2}}} \\
& \leqq \frac{1}{(\cos \beta / 2)\left|\sqrt{w_{1}}\right|+(\cos \beta / 2)\left|\sqrt{w_{2}}\right|} \\
& \leqq \frac{1}{(\cos \beta / 2)(2 \sqrt{0.5})} \leqq 0.87=r<1
\end{aligned}
$$

Since the diameter of $R_{d}$ is $2 d$ and $r<1$, the lemma is true for $n \leqq 3$. To obtain the result for larger $n$, we use (1) and then apply (2) repeatedly. Q.E.D.

Theorem 9. Let $0<\lambda<0.2, z \in R_{d}$, and $d \geqq a$. Then $S(\omega)$ exists and lies in $R_{a}$, for all $\omega \in \Omega$, and

$$
\left|S_{n}(\omega, z)-S(\omega)\right|<2 d(0.87)^{n-3}
$$

Proof. Let $m \geqq n$ and note that

$$
\left|S_{n}(\omega, z)-S_{m}(\omega, z)\right|=\left|S_{n}(\omega, z)-S_{n}\left(\omega, S_{m-n}(\tilde{\omega}, z)\right)\right|
$$

for some $\tilde{\omega} \in \Omega$. Moreover $S_{m-n}(\tilde{\omega}, z) \in R_{d}$ by Lemma 6 . Hence Lemma 8 applies with $z_{1}=z$ and $z_{2}=S_{m-n}(\tilde{\omega}, z)$, yielding

$$
\left|S_{n}(\omega, z)-S_{m}(\omega, z)\right| \leqq 2 d r^{n-3} .
$$


Thus $\left\{S_{n}(\omega, z)\right\}_{n=1}^{\infty}$ is a Cauchy sequence in the closed set $R_{d}$, and by Lemma 8 the limit is independent of $z \in R_{d}$. If we take $z \in R_{a}$ we find by Lemma 6 the limit $S(\omega)$ also belongs to $R_{a}$. Q.E.D.

Next we relate $S(\omega)$ to $F_{\lambda}$ and $\left\{f_{n}\right\}$.

Theorem 10. Let $0<\lambda<0.2$, and let $\theta$ and $\omega$ be related as in Theorem 7 . Then

and

$$
\operatorname{Lim}_{n \rightarrow \infty} f_{n}\left(e^{i \theta}\right)=S(\omega)
$$

$$
\operatorname{Lim}_{\substack{z \rightarrow e^{\imath \theta} \\|z|>1}} F_{\lambda}(z)=S(\omega)
$$

Proof. Notice that for some values of $\theta$ there are two expansions $\theta=2 \pi \sum_{j=1}^{\infty} d_{j} / 2^{j}$, one involving infinitely many zeros and one involving infinitely many ones. In these cases there correspond two distinct elements $\omega_{1}$ and $\omega_{2}$ in $\Omega$, and for the theorem to make sense it must be true that $S\left(\omega_{1}\right)=S\left(\omega_{2}\right)$. But this is just what is implied by Lemma 7. Accordingly, without loss of generality, we can assume that $\omega$ contains infinitely many +1 's.

Let $z=\Gamma e^{i \theta}$. Then it follows that $0 \leqq \arg z^{2^{-1}}<\pi$ precisely when $d_{j}=0$. Using the definition of $f_{n}$ we obtain

$$
f_{n}(z)=S_{n}\left(\omega, f_{0}\left(z^{2 n}\right)\right) \quad \text { for } \quad \Gamma \geqq 1 .
$$

If $\Gamma=1$ then $f_{0}\left(z^{2^{n}}\right) \in R_{a}$ and we can let $n \rightarrow \infty$ with the aid of Theorem 9, yielding (3).

To obtain (4), first let $\varepsilon>0$ and choose $d=2 a$ in Lemma 8. Pick $n$ so that $4 a(0.87)^{n-3}<\varepsilon$. Since $B_{\lambda} \subset R_{a}$ there exists $\varrho>1$ such that

$$
F_{\lambda}(z) \in R_{2 a} \text { for } 1<|z|<\varrho .
$$

Let $I=\left\{\phi \mid \frac{\phi}{2 \pi}-\frac{1}{2^{n+1}}<\sum_{j=1}^{n} \frac{d_{j}}{2^{j}} \leqq \frac{\phi}{2 \pi}\right\}$. From the Böttcher equation (1b) we find that

$$
F_{\lambda}\left(\Gamma e^{i \phi}\right)=S_{n}\left(\omega, F_{\lambda}\left(\Gamma^{2^{n}} e^{2^{n} i \phi}\right)\right) \quad \text { for } \quad \phi \in I .
$$

Combining this with (5) and Theorem 9 we obtain

$$
\left|F_{\lambda}\left(\Gamma e^{i \phi}\right)-S(\omega)\right|<\varepsilon \text { for } \phi \in I \text {, and } 1<\Gamma<\varrho^{1 / 2^{n}} .
$$

If $\theta$ is an interior element of $I$ we are done. Otherwise $\theta$ is the left endpoint and $\theta / 2 \pi$ has a second binary representation which we can use similarly to show we can allow $\phi<\theta$ in (6). Q.E.D.

Next we extend the allowed values of $\lambda$ from the interval $0<\lambda<0.2$ to the set $L=\{\lambda \in \mathbb{C}|| \lambda \mid<1 / 4\}$. Observe that the inductive definition of $\left\{f_{n}\right\}$ of Lemma 3 applies also for $\lambda \in L$, and that we can establish the containment condition

$$
\{z \in \mathbb{C}|| z \mid>C\} \supset f_{n}\left(D_{0}\right) \supset f_{n-1}\left(D_{0}\right)
$$

for $n \in\{1,2, \ldots\}$. As before, we prove the existence of the limit $F_{\lambda}$ of the sequence $\left\{f_{n}\right\}$, and thus we obtain direct proof of the existence of a conformal mapping of $D_{0}$ which solves the Böttcher equation (1b). 
In the following theorem we alter the notation slightly to emphasize the dependence upon $\lambda$.

Theorem 11. For any fixed $\lambda \in L$ let $F(\lambda, z)$ be the conformal mapping of $D_{0}$ which solves the Böttcher equation (1b). Then $F$ may be extended continuously to $L \times \overline{D_{0}}$, where it is analytic in $\lambda$. The functions $G_{\lambda}(\theta)=F\left(\lambda, e^{i \theta}\right)$ are uniformly Hölder continuous for $\lambda$ in any compact subset of $L$.

Proof. Let $f_{n}(\lambda, z)=f_{n}(z)$. Observe that for $\lambda \in L$ and $z \in \overline{D_{0}}$ the functions in $\left\{f_{n}(\lambda, z)\right\}$ as functions of $\lambda$ are analytic, and their images omit the disk $\{w \in \mathbb{C}|| w \mid<C\}$. Hence they constitute a normal family. This means that they have an infinite subsequence which converges to some function $H(\lambda, z)$ which, among its other properties, is analytic in $\lambda$ for $\lambda \in L$, for each fixed $z \in \bar{D}_{0}$. Suppose that there are in fact two different subsequences convergent to two different functions $H_{1}$ and $H_{2}$. Since the whole sequence is convergent to a single limit for $0<\lambda<0.2, H_{1}$ and $H_{2}$ agree on a set which contains a limit point. Hence by Vitali's theorem $H_{1} \equiv H_{2}$. Thus, we have the existence of

$$
H(\lambda, z)=\operatorname{Lim}_{n \rightarrow \infty} f_{n}(\lambda, z)
$$

analytic in $\lambda$ for $\lambda \in L$ for each fixed $z \in \bar{D}_{0}$. Moreover $H(\lambda, z)=F(\lambda, z)$ for $z \in D_{0}$. Next consider $\operatorname{Lim}_{\substack{z \rightarrow e^{i \theta} \\|z|>1}} F(\lambda, z)$. For $0<\lambda<0.2$ this limit exists and equals

$\operatorname{Lim}_{n \rightarrow \infty} f_{n}\left(\lambda, e^{i \theta}\right)=H\left(\lambda, e^{i \theta}\right)$. Thus, by again applying Vitali's theorem, we have that the limit exists for all $\lambda \in L$ and

$$
\operatorname{Lim}_{\substack{z \rightarrow e^{i \theta} \\|z|>1}} F(\lambda, z)=\operatorname{Lim}_{n \rightarrow \infty} f_{n}\left(\lambda, e^{i \theta}\right)
$$

It now follows that as a function of $z, F(\lambda, z)$ is a one-to-one analytic function on $D_{0}$ with well-defined boundary values.

Finally, we establish the Hölder continuity. Since $C>1 / 4$ for all $\lambda \in L$ we can choose $\beta$ with $0<\beta<\frac{\pi}{2}$ and $R>1$ such that for any compact subset $M$ of $L$

$$
2 \sqrt{C} \cos (\beta / 2)>R>1 \text { for all } \lambda \in M \text {. }
$$

Since $G_{\lambda}(\theta)$ is continuous in $\lambda$ and $\theta$ we can choose $\gamma>0$ such that

$$
\left|\arg \left(G_{\lambda}(\theta) / G_{\lambda}(\phi)\right)\right|<\beta \quad \text { if } \quad|\theta-\phi|<\gamma \quad \text { with } \quad \lambda \in M .
$$

Let $\theta$ and $\phi$ be real with $|\theta-\phi|<\gamma$. Then there exists an integer $n \geqq 0$ such that

$$
\gamma / 2^{n+1} \leqq|\theta-\phi|<\gamma / 2^{n} \text {. }
$$

Hence

$$
\begin{aligned}
\left|G_{\lambda}(\theta)-G_{\lambda}(\phi)\right| & =\left|\sqrt{G_{\lambda}(2 \theta)}-\sqrt{G_{\lambda}(2 \phi)}\right| \\
& =\frac{\left|G_{\lambda}(2 \theta)-G_{\lambda}(2 \phi)\right|}{\left|\sqrt{G_{\lambda}(2 \theta)}+\sqrt{G_{\lambda}(2 \phi)}\right|} \\
& \leqq \frac{\left|G_{\lambda}(2 \theta)-G_{\lambda}(2 \phi)\right|}{R} .
\end{aligned}
$$



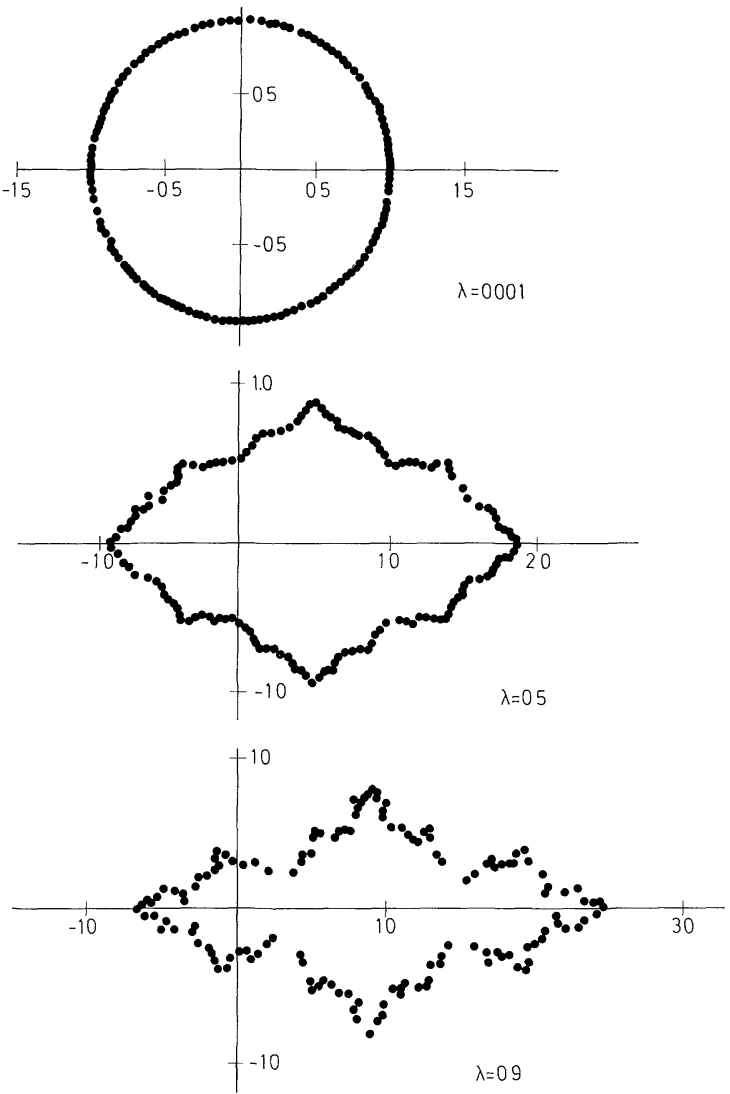

Fig. 3. Representation of $B_{\lambda}$ for $\lambda=0.001,0.5$, and 0.9 . The crosses indicate dots in the complex plane which belong to $B_{\lambda}$. These drawings are based upon accurate plots of five hundred points in $B_{\lambda}$, picked at random

Iterating we find

$$
\left|G_{\lambda}(\theta)-G_{\lambda}(\phi)\right| \leqq \frac{\left|G_{\lambda}\left(2^{n} \theta\right)-G_{\lambda}\left(2^{n} \phi\right)\right|}{R^{n}} \leqq \frac{K_{1}}{R^{n}}
$$

where $K_{1}$ is the maximum diameter of $B_{\lambda}$ for $\lambda \in M$. Using (7), with $\alpha=\ln R / \ln 2$ and $K_{2}=K_{1}(2 / \gamma)$ we have

$$
\left|G_{\lambda}(\theta)-G_{\lambda}(\phi)\right| \leqq K_{2}|\theta-\phi|^{\alpha} \quad \text { for } \quad|\theta-\phi|<\gamma .
$$

To allow for $|\theta-\phi| \geqq \gamma$ we replace $K_{2}$ by

$$
K=\operatorname{Max}\left\{K_{2}, K_{1} \gamma^{-\alpha}\right\} \text {. Q.E.D. }
$$

We can illustrate some of the boundary behavior by calculating $\frac{\partial F}{\partial \lambda}(0, z)$. We use the fact that $\partial F / \partial \lambda=\operatorname{Lim}_{n \rightarrow \infty} \partial f_{n} / \partial \lambda$ and, for $n>0$,

$$
\frac{\partial f_{n}}{\partial \lambda}(\lambda, z)=1+\frac{1}{\sqrt{f_{n-1}\left(z^{2}\right)}} \cdot \frac{1}{2}\left(1+\frac{1}{\sqrt{f_{n-2}\left(z^{4}\right)}} \frac{1}{2}\left(1+\ldots\left(1+\frac{1}{2} \frac{\sqrt{b}}{\sqrt{f_{0}\left(z^{2 n}\right)}}\right) \ldots\right)\right) .
$$



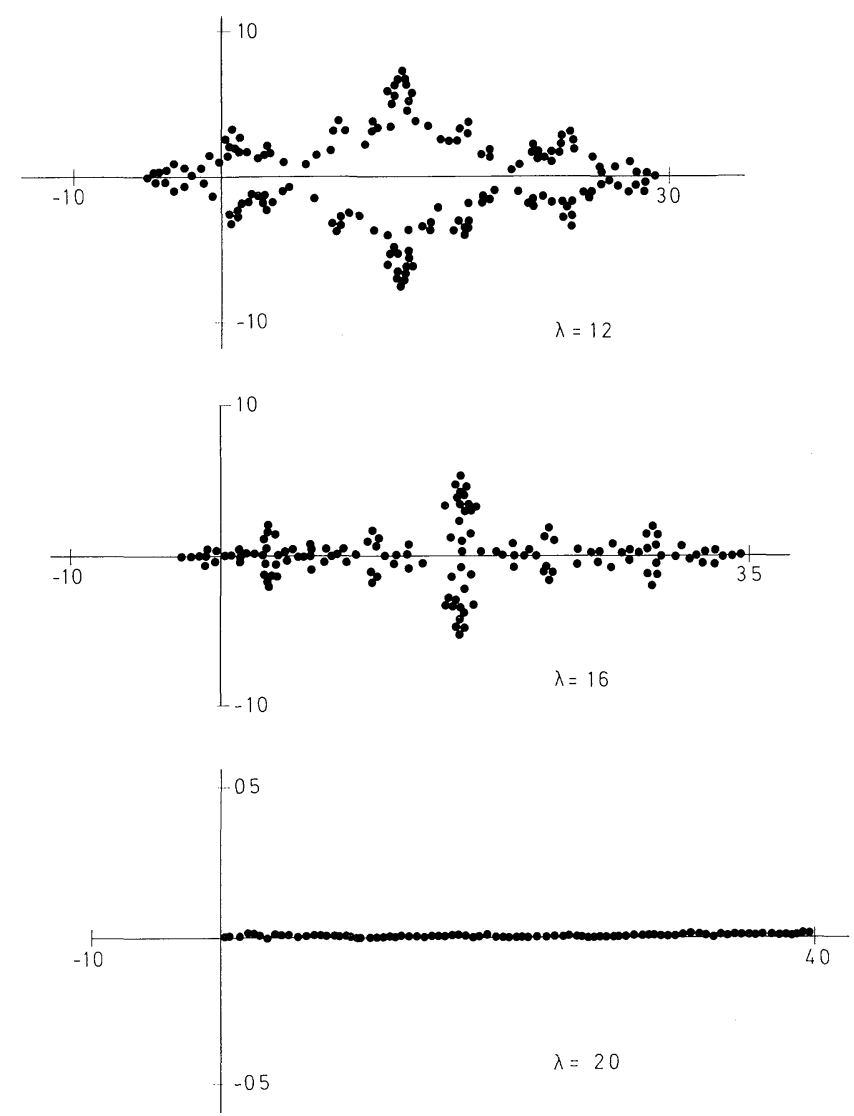

Fig. 4. Representation of $B_{\lambda}$ for $\lambda=1.2,1.6$, and 2.0. See the caption of Fig. 3

Then since $f_{n}(0, z)=f(0, z)=z$ (note that $b=1$ when $\left.\lambda=0\right)$ it follows that

$$
\frac{\partial F}{\partial \lambda}(0, z)=1+\sum_{j=1}^{\infty} \frac{1}{2^{j} z^{2^{j}-1}} .
$$

This series implies just the expected boundary behavior. It converges uniformly for $|z| \geqq 1$ and is analytic for $|z|>1$. On the other hand, it is a gap series and thus the unit circle is the natural boundary of the domain of the analytic function. In particular, we find that

$$
\operatorname{Lim}_{\Gamma \rightarrow 1+} \frac{\partial^{2} F}{\partial z \partial \lambda}\left(0, \Gamma e^{i \theta}\right)=\infty \text { for } \theta=2 \pi / 2^{n}
$$

\section{Pictures Relating to $B_{\lambda}$}

Here we present pictorially the results of some calculations which concern the structure of $B_{\lambda}$.

Figures 3 and 4 represent $B_{\lambda}$ for $0 \leqq \lambda \leqq 2$. (See also [21] where better pictures are given.) These drawings are not exact, but are based upon similar ones in which 


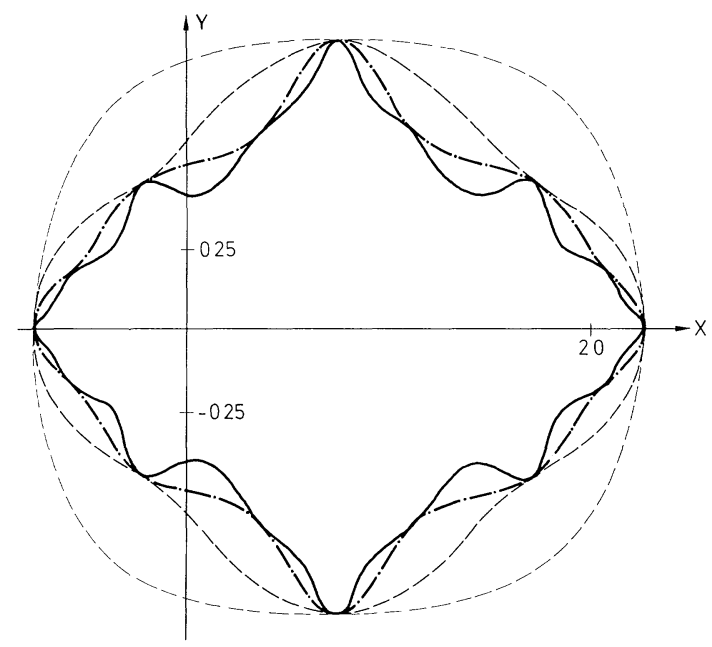

Fig. 5. Successive inclusion domains for $B_{\lambda}$ when $\lambda=0.75$. The boundary indicated by $n \in\{1,2,3,4\}$ is the image of the unit circle under $f_{n}(x)$. The sequence of boundaries converges to $B$, in the manner described in Sect. 3.1

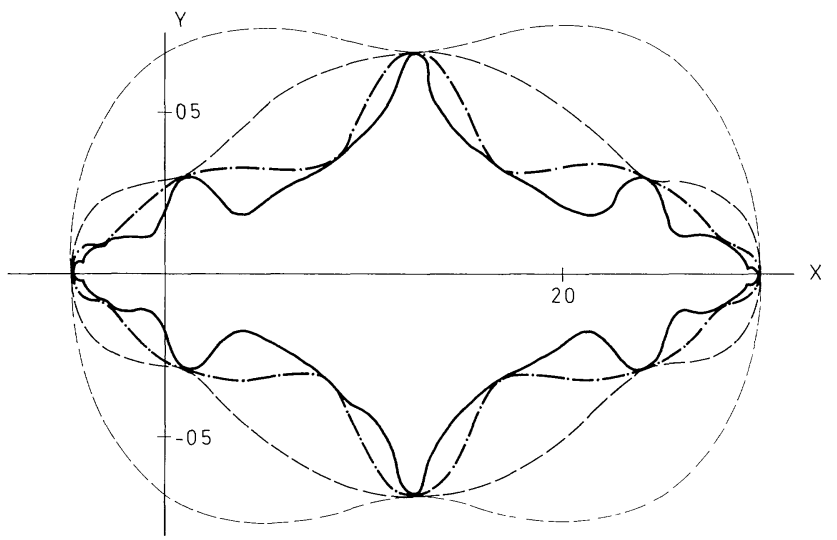

Fig. 6. Same as Fig. 5 , but here $\lambda=1.25$

about five hundred points in $B_{\lambda}$ were plotted. The points were all of the form $S_{50}(\omega, a)$, and $\omega \in \Omega$ was chosen at random.

An alternative view of $B_{\lambda}$ is in Figs. 5 and 6 where we have plotted the boundaries of the sets $f_{n}\left(D_{0}\right)$ for $n \in\{1,2,3,4\}$, at two different values of $\lambda$. Recall that $f_{1}\left(D_{0}\right) \subset f_{2}\left(D_{0}\right) \subset f_{3}\left(D_{0}\right) \subset \ldots$, and that the boundary of the set obtained in the limit is $B_{\lambda}$.

Acknowledgements. We thank D. Ruelle for telling us the references to Fatou, Julia, Brolin, and Mandlebrot. The original motivation for our approach, the search for a meaning for the set $\tilde{B}_{\lambda}$, was research on the diophantine moment problem [3]. We also thank O. Lanford for his help in the revision of this work. 


\section{References}

1. Adler, R.L., Rivlin, T.J.: Ergodic and mixing properties of Chebyshev polynomials. Proc. Am. Math. Soc. 15, 794-796 (1964)

2. Baker, G.A., Jr.: The essentials of Padé approximants. New York: Academic Press 1978

3. Barnsley, M.F., Bessis, D., Moussa, P.: The diophantine moment problem and the analytic structure in the activity of the ferromagnetic Ising model. J. Math. Phys. 20, 535-545 (1979)

4. Barnsley, M.F., Geronimo, J.S., Harrington, A.N. : Some treelike Julia sets and Padé approximants. Submitted to Letters in Math. Phys. (1982)

5. Barnsley, M.F., Geronimo, J.S., Harrington, A.N.: Orthogonal polynomials associated with invariant measures on Julia sets. Bull. Am. Math. Soc. 7, 381-384 (1982)

6. Billingsley, P.: Ergodic theory and information. New York: Wiley 1965

7. Blumenthal, O.: Über die Entwicklung einer willkürlichen Funktion nach den Nennern des Kettenbruches für $\int_{-\infty}^{+\infty} \frac{\phi(\varepsilon)}{(z-\varepsilon)} d \varepsilon$. Inaugural dissertation. Göttingen 1898

8. Brolin, H. : Invariant sets under iteration of rational functions. Ark. Mat. 6, 103-144 (1965)

9. Chihara, T.S.: An introduction to orthogonal polynomials. New York: Gordon and Breach 1978

10. Collet, P., Eckmann, J.: Iterated maps on the interval as dynamical systems. Basel, Boston: Birkhäuser 1980

11. Douady, A., Hubbard, J.H.: Itérations des polynômes quadratiques complexes, C. R. Acad. Sci. Paris, Sér. I Math. 294, 123-126 (1982)

12. Fatou, M.P.: Sur les équations fonctionnelles. Bull. Soc. Math. France 47, 161-271; 48, 33-94; 48, 208-314 (1919)

13. Feigenbaum, M.: Quantitative universality for a class of nonlinear transformations. J. Stat. Phys. 19, 25-52 (1978)

14. Geronimo, J.S., Case, K.N. : Scattering theory on polynomials orthogonal on the real line. Trans. Am. Math. Soc. 258, 467-494 (1980)

15. Goluzin, G.M.: Geometric theory of functions of a complex variable. In: Translations of Mathematical Monographs, Vol. 26. Providence, RI: American Mathematical Society 1969

16. Jakobson, M.V.: Absolutely continuous invariant measures for one-parameter families of onedimensional maps. Commun. Math. Phys. 81, 39-88 (1981)

17. Hille, E.: Analytic function theory, Vol. II. New York: Chelsea Publishing Company 1962

18. Hurewicz, W., Wallman, H. : Dimension theory. Princeton: Princeton University Press 1948

19. Julia, G.: Mémoire sur l'iteration des fonctions rationnelles. J. Math. Pures Appl. 4, 47-245 (1918)

20. Mandlebrot, B.: Fractals: form, chance, and dimension. San Francisco: W. H. Freeman, 1977. Also a forthcoming book, from the same publisher

21. Mandlebrot, B.: Fractal aspects of the iteration of $z \rightarrow \lambda z(1-z)$ for complex $\lambda$ and $z$. Ann. N.Y. Acad. Sci. 357, 249-259 (1980)

22. Szego, G.: Orthogonal polynomials. American Mathematical Society Colloquium Publication No. 23. Providence, RI : American Mathematical Society 1939

23. Ulam, S., von Neumann, J.: On combination of stochastic and deterministic processes. Bull. Am. Math. Soc. 53, 1120 (1947)

Communicated by O. E. Lanford

Received February 18, 1982; in revised form September 20, 1982 
